\title{
Critique and Comparison of Prevailing Consciousness Models with a Novel Embodied Cognition Model
}

\author{
Ravinder Jerath*, Connor Beveridge \\ Charitable Medical Healthcare Foundation, Augusta, GA, USA \\ Email: ^Rj605r@aol.com
}

How to cite this paper: Jerath, R. and Beveridge, C. (2018) Critique and Comparison of Prevailing Consciousness Models with a Novel Embodied Cognition Model. World Journal of Neuroscience, 8, 370-401. https://doi.org/10.4236/wjns.2018.83030

Received: August 9, 2018

Accepted: August 28, 2018

Published: August 31, 2018

Copyright (c) 2018 by authors and Scientific Research Publishing Inc. This work is licensed under the Creative Commons Attribution International License (CC BY 4.0).

http://creativecommons.org/licenses/by/4.0/

\section{(c) (i) Open Access}

\begin{abstract}
In order to produce insight to the current state of consciousness research, we provide a review of some of the more preeminent models of consciousness via a comparison of these theories with a novel holistic model. The main goal of this review is to present the advantages in theoretical power of an embodied cognition, metastable model of consciousness manifest in the Default Space Theory by comparing it to other popular brain-based metastable models that though provide important insight into the cognitive nature of consciousness, are incomplete in their model of an architecture of consciousness. The Default Space Theory is a world simulation model in which the thalamus fills in processed sensory information from corticothalamic feedback loops into an internal 3D matrix termed the default space. This article examines some of the most regarded brain-based, metastable consciousness models, the Operational Architectonics Theory of Brain-Mind, Global Workspace Theory, Information Integration Theory, and the Dynamic Core Hypothesis. Through an overview and critique of these models, we illustrate their insights and deficits, and discuss why the Default Space Theory is the most comprehensive theory of consciousness.
\end{abstract}

\section{Keywords}

Default Space, Metastability, Consciousness, Information Integration, Global Workspace

\section{Introduction}

Consciousness can be defined simply in terms of the presence of phenomenal experience. This can be considered a valid definition, as we all are aware when we are having an experience versus being unaware of anything as in deep sleep. 
Tononi also defines consciousness as everything we experience [1]. Edelman and Baars define consciousness in vertebrates as a dynamic, integrated, multimodal process necessitated by physical activity in the forebrain [2]. Although it may be difficult to define, we all have our own intuitions about what it is. There are certainly aspects of human consciousness that are difficult to deny that are proposed by Tononi and are accounted for in the Default Space Theory (DST) [1]. It is established via common experience that consciousness is unified into a singular whole, structured, certainly exists, and is phenomenologically distinct in its composition [1]. While experiences are entirely subjective, meaning we cannot directly experience another person's perspective, they are somewhat predictable. We can imagine what another is experiencing through our ability of theory of mind communicated by facial expressions, gestures, behaviors, and verbal reports [3]. While the phenomenon of consciousness is subjective in this way, there must be an objective source for its existence. This objectivity surely lies in some natural, physical processes and the mystery of this process leads us to the hard problem of consciousness [4], quite possibly the final frontier of science. Whether consciousness serves a functional purpose or simply emerges from functional biological processes as an epiphenomenon is its own mystery [4].

When it comes to neuroscience, metastable theories of consciousness propound that consciousness is based upon the global integration of separate functional parts that while maintaining their own independent processing activities, coordinate and cooperate together via oscillations and reentrant activity [5]. Metastability therefore harmonizes the tendency of brain regions to express their own autonomy and the tendency for those regions to form synergy on a global and macroscopic scale [6] [7]. In the metastable brain, local and global activities coexist in complement, not in confliction, at the same time. This dynamic, self-assembling process of different brain modules engaging and disengaging in time occurs via the binding of distributed and independent oscillations into coherent networks [8] [9] [10]. Metastable models propose that this cooperative mechanism is responsible for the "binding problem" of how different perceptual features of a scene and even the vast number of features of one's conscious experience are bound together into a singular coherent entity [11]. Coupling between distal functional modules has been demonstrated by long-range functional connectivity [12].

Traditional views on cognition have taken a brain-based stance, assuming the existence of internal representations generated and maintained by distinct and specific mechanisms in the brain [13]. Emotions have also been posited to be localized to specific and dedicated networks in the brain such as the brainstem and limbic structures [14]. Cognitive faculties such as learning, reasoning, and memory have been associated with more highly evolved cortical sites [15]. The computational theory of mind holds that the brain is an information processing system and that cognition, including consciousness, is a form of computation [16]. This view that the mind is generated by neural activity in the brain is the 
most common in cognitive psychology, and many cognitive architectures that attempt to explain consciousness such as the Global Workspace Theory take this approach. According to this brain-based view, the body is simply a tool for delivering input derived from the world and acting on output from activity in the brain.

In an extension to brain-based cognition, embodied cognition is a radical hypothesis that proposes the brain is not the only source of cognitive functioning, instead, our bodies also play a significant role in cognition [17]. This hypothesis is radical in that it is new to cognitive science and rejects the common brain-based treatment of cognition [18]. Although the field is not unified in its established definitions [17], the most accepted and common view is that cognition is deeply dependent on the body, and the body acts as a constrainer of cognitive representations, a distributor of computational and representational load, and a regulator of cognitive activity, coordinating cognition and action [13]. In this way, our bodies do much of the cognitive work in pursuing goals, thus replacing the need for complex internal mental representations [17]. Evidence is growing in support for an embodied cognitive approach, demonstrating that cognition is highly dependent on sensory-motor processes [19]. Thus, sensing and acting should be considered as a significant component of thinking itself. For instance, forced laughter and smiling has been shown to substantially improve mood in participants [20]. Through the DST, we extend the embodied cognitive approach to consciousness in that the brain, mind, and body act as a single entity in a holistic manner in creating consciousness.

\section{The Default Space Theory}

The DST provides a body-wide bioelectric cognitive architecture which gives rise to our unified human experience, thus providing a physical complement to the psychical nature of consciousness [21]. The "substance" of this bioelectric structure consists of layers of electrical activity throughout the body, although mostly in the brain. The base of this layered hierarchy is the electric activity of single neurons, cells, and synapses. The action potentials and membrane potential oscillations of cells are the building block of higher order bioelectric functions which form the activity giving rise to consciousness. These microscopic events do not directly result in cognition or experience [22]. The middle of this hierarchy consists of coordinated electrical activity of local neural assemblies which can be detected by electroencephalography [23]. This level begins to support cognition and is some cases consciousness. The upper level of this hierarchy is composed of electric oscillatory synchrony among many of the local neural assemblies. Finally, at the peak of this layered hierarchy, these distributed local oscillatory synchronies can be synchronized together on a global scale leading to the unified experience. We consider this upper layer higher order due to its complexity and integrated nature. Thus, we propose these properties and the global unification of the bioelectric oscillations are the physical correlates of 
consciousness.

This architecture generates an internal, subconscious, virtual, three-dimensional (3D) space-time matrix we have termed the default space which houses a conscious simulation of the external environment [21] [24]. We term this the default space because it is a virtual space that defaults to the qualities of the external world, replicating its dimensions of space and time. Thus, "default" indicates an automatic state of the phenomenology of consciousness. The existence of this space is illustrated in the condition of contralateral neglect described later in the article. It is within this internal space that all sensory perceptions are bound [24]. Although these perceptions are dominated by top-down cognitive functions, sensory receptors are synchronized with the qualia of this space and are part of its bioelectric framework. For instance in the case of vision, photoreceptors synchronize with relevant cortical areas which creates a framework of the visual aspect of the default space. The resulting simulation is micro-calibrated with the physiology of the retina, and [25], through this mechanism, light stimuli that fall upon the retina are automatically bound in size, location, color and movement to current objects in conscious awareness which are themselves bound to the spatial dimension of the default space. In this way, all sensory qualia can be unified, integrated, and experienced as one whole. This unification and integration is coordinated by the thalamus via corticothalamic feedback loops [24]. The role of the thalamus in consciousness and sensory processing is supported by a growing body of research [26] [27] [28].

All conscious experience occurs within the default space and its spatial aspect is essential. Dreams, recollection of memories, and hallucinations all occur within this space and have a spatial foundation [29]. Evolutionary origins for the default space are derived from the replication of the external physical environment within. This provides the evolutionary benefits of optimal perception of and survival in such an environment. The default space is maintained by the harmonic oscillatory activity of membrane potentials from all cells of the body [29]. This large electro-chemical space is "observed" in respect to the thalamus [24]. Even when lacking sensory stimuli or internally generated sensation, the default space remains in an idling state. We have proposed how this structure of an empty 3D matrix is maintained by baseline neural activity such as that from the default mode network and brainstem [30]. Even in this idling state, the sensory organs are synchronized with the relevant cortical areas. The many various cortical areas are themselves synchronized with each other via coordination of membrane potential oscillations by the thalamus. This metastable theoretical basis of the DST describes how the many different forms of sensation are integrated into a unified whole.

Through the phenomenon of lateral inhibition, cortical sites modulate sensory organs and are synchronized with them via oscillatory communication, mostly in the gamma frequency [31]. Through this communication, corticothalamic networks interface with sensory receptors which allow for near instant information transfer [32]. We use the term lateral inhibition in a different manner than 
the commonly accepted terminology. Instead of this information transfer being local, lateral, and adjacent through physical neighboring, the sensory receptors are "adjacent" to the corticothalamic networks via oscillatory synchronization. Because the speed of charge transmission is so fast, it is as if the physical separation is non-existent. This synchronization allows sensory processing to occur at the sight of the sensory organ and for the sensory organs to anticipate expected sensory stimuli [31]. These expectations can lead to magnification of certain sensory information and filtering of unwanted data [31]. The synchronization of the sensory receptors with the current state of the simulation leads to a seamless flow of sensory experience. By maintaining the contents of sensory experience at the sensory receptors themselves, the need for complex mental representations of the contents of experience can be replaced by physical representations at the site of the sensory receptors themselves.

\section{Overviews of Leading Metastable Models of Consciousness}

The brain dynamic of metastability has recently become recognized as likely the most important system for understanding the nature of consciousness [6]. There exists a variety of metastable models of consciousness that attempt to associate biological processes to the physiological and phenomenological characteristics of consciousness by citing the global integration of independent networks. The models we explore are some of the most prominent in the field of cognitive neuroscience of consciousness and although they do provide great insight into cognition, we assert that they are incomplete in the scope of its mechanisms. No consciousness model has been widely accepted as conclusive or even as a foundation for such a conclusive model. These models attempt to tackle the real problem of consciousness, how to account for the properties of consciousness in terms of biological mechanisms [33]. These models are all brain-based in that they propose consciousness arises from processes solely occurring within the brain, specifically the cortex and thalamus.

\subsection{Operational Architectonics Theory of Brain-Mind}

Operational Architectonics Theory (OAT) developed by the Fingelkurts twins is centered upon metastable brain states creating a complex, hierarchical, bioelectric architecture that is the framework for conscious and subconscious mental states which allows for the complementation and coordination of brain and mind phenomena in space and time [11] [34]. OAT identifies this brain-mind complementation as a functional isomorphism between the neurophysiological, bioelectric, oscillatory framework termed operational space-time, and the framework of conscious experience termed phenomenal space-time [11]. This phenomenal space is viewed by the Fingelkurts as the fundamental ingredient in the phenomenon of mind and consciousness which is a unifying, spatial, 3D coordinate system [11]. This empty 3D matrix, or "virtual space", is subcons- 
cious, however, all conscious contents must be embedded in it in order to be present in one's subjective experience [11].

OAT describes a hierarchy of neuronal activity that corresponds to a hierarchical level of consciousness [11]. The top level being our reflective conscious awareness is an integrated experience created from the integration of complex macro-operations which are themselves integrations of independent neuronal assemblies [11]. These neuronal assemblies support elementary cognitive operations and are therefore considered by the Fingelkurts to be conscious or unconscious, while the basic level of individual neurons is considered nonconscious [11]. The top level of the hierarchy is therefore heavily separated from the basic physical activity of neurons which creates the strangeness of consciousness and the inability to tie it directly to the chemistry and physics of neurons themselves [35]. The Fingelkurts argue that the vast neural network of the brain and the resulting hierarchically higher level of dynamic electromagnetic fields constitute the subconscious 3D matrix described as the foundational architecture of consciousness [11].

The subconscious 3D space-time matrix is argued to be a mediator between the purely neurophysiological domain and the domain of conscious phenomena [11]. The Fingelkurts identify the spatially and temporally structured electromagnetic field of the brain as the candidate for which conscious phenomena can be tied [36]. They support this correlation in part through studies showing local bioelectric fields are correlated with conscious percepts [37] [38]. Metastable spatial and temporal patterns in the electromagnetic fields identified as operational modules $(\mathrm{OM})$ result from synchronous activity between spatially distributed neural assemblies and constitute a higher level of abstractness compared to the underlying anatomical neurophysiology [34]. Several OMs can coexist at the same time and with certain subsets of neural assemblies belonging to multiple OMs [11]. The Fingelkurts propound that the integrated experience of consciousness could result from independent OMs further synchronized between each other resulting in a more complex and abstract OM [35] (Figure 1). At this level, the electrical framework is functionally isomorphic with the organization and phenomenal quality of consciousness [11]. Synchronization of processes by different neural assemblies located in different brain regions allows the binding of dispersed sensory features into integrated patterns of qualities and ultimately into a unified complex scene [35].

\subsection{Global Workspace Theory}

One of the currently most popular models of consciousness beginning development by Bernard Baars in 1982, Global Workspace Theory (GWT), has foundations in the earliest days of artificial intelligence from a concept of "blackboard architecture" [40]. This term refers to a design pattern that allows collaborative coordination of various individual programs which cooperatively solve problems no individual program could solve on its own [41]. GWT takes into account the 
A



C PHYSICAL TIME ARROW

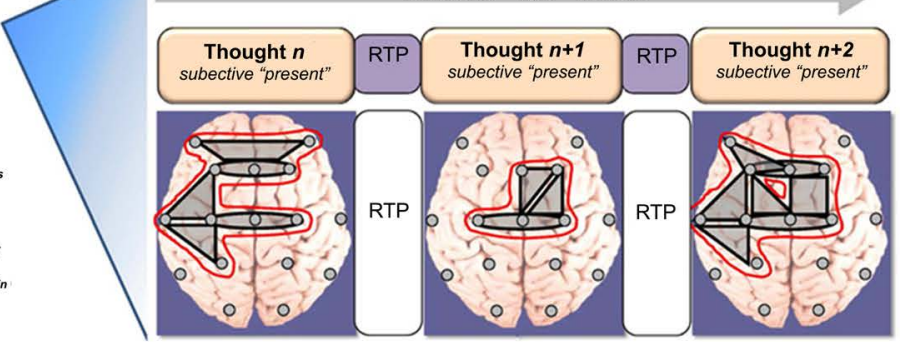

Figure 1. Operational Architectonics Concept of a Functional hierarchy of Neuronal Bioelectric Fields and Operational Modules Producing Consciousness. A) The hierarchy of bioelectric operations consists of higher levels composed of synchrony among lower levels. The lowest level contains oscillatory activity arising from independent neural assemblies. The middle level contains OMs, which consist of a synchrony among electrical activity from dispersed assemblies. The highest level consists of complex OMs which are synchronies of distinct synchronized activity; B) An illustration of an individual neuronal assembly's dynamic waveform activity which contains multiple segments in time divided by rapid transitional processes (RTPs); C) OMs are illustrated spatially which span varying brain areas. Synchronies among the various regions can change dynamically resulting in distinct phenomena of consciousness. The grey shapes are simple OMs which synchronize together forming a more abstract, upper level $\mathrm{OM}$ (outlined in red) which are responsible for the unified, integrated experience (Permission for image use provided by Creative Commons Licensing. Image obtained from [39] published by Frontiers).

limited nature of consciousness in comparison to the vast capabilities of the unconscious mind [42]. In doing so it identifies the mind as a "great society" of unconscious parallel neural nets, layers, and connections conjoined with a cognitively efficient but limited conscious component [42] [43]. The theory's main proposition is that the distinct neural events that cross a certain activity threshold become globally available, and it is this information that is identical to conscious experience [42]. Thus, the functional role of consciousness is to provide various types of functional access between varying mental functions such as executive functions, sensation, intention, voluntary action, and learning [44].

A summarized view of the theory is given as a theater metaphor. In this perspective, the full stage of the theater corresponds to our working memory [42]. Focal consciousness is the illuminated scope from a spotlight pointed at the stage of the theater of mind [45]. In this spotlight of the stage is the "workspace" which corresponds neurologically to not one anatomical hub, but to a functional hub that signals, binds, and propagates information over multiple unconscious networks [46]. The unconscious mind consists of modular processing units that when activated sufficiently cross the threshold into this functional hub where the information becomes available for global processing [46]. This functional hub is propounded by Baars to be the corticothalamic system which permits constant bidirectional signaling between many cortical areas [46]. This thalmocortical network can be metaphorically understood as the world-wide web of the brain 
[46].

A foundation of Baars' theory is the ability to acquire conscious control and/or awareness over the vast unconscious domain. It is this global access of the spotlight of consciousness awareness/control in the unconscious, "dark", theater of mind that allows certain "actors" to have their light on the theater stage which is the "workspace" of a play. The "behind the scenes" activities and plans of the playwright, director, scene setters, and audience are the unconscious systems that shape the conscious events on the stage [42]. Thus, there is a convergence of input from the acting coaches, scene designers, and so on that lead to the conscious event of the actors performance in the spotlight of the stage. Once a word is spoken however, the message is "broadcast" globally to the audience and director backstage where they use it to perfect the next performance. The audience plays the unconscious role of demanding what they want to see on stage, as well as doing problem solving and knowledge banking themselves. This phenomenon of questions presented to consciousness and resulting in an unconscious reveal of answers could be seen in an open forum theater setting. When a person on stage asks a question, appropriate audience members may walk on stage and speak the answer in the spotlight [42].

\subsection{Integrated Information Theory}

Integrated Information theory (IIT), currently in its third version IIT 3.0, associates consciousness to physical systems that integrate information [47], thus posing an answer for the hard problem of consciousness. This theory initially proposed by Tononi in 2004 propounds a mathematical model of consciousness based on its phenomenology and how physical systems can account for these phenomena, proposing a way to mathematically measure its quantity and quality. It proposes a numerical value in measuring the quantity of consciousness, $\Phi$, which indicates the amount of information that can be integrated by an irreducible system [1]. The term irreducible indicates a gestalt concept that the system generates information above and beyond the information generated by its parts [47]. If it can be reduced in components in anyway and still contains the same cause-effect repertoire, it is not irreducible. According to IIT, consciousness is an intrinsic property of the universe much like mass, charge, or spin. Therefore, consciousness doesn't have a functional purpose in and of itself, but is an epiphenomenon arising from systems which integrate information which is a very useful mechanism evolutionarily speaking.

This theory is unlike the many others in that instead of founding its concepts on neural mechanisms of consciousness and then working towards its physical mechanisms, it initially tries to explain the fundamental physical origins of experience in the universe. According to IIT, at its fundamental level, consciousness is integrated information [48]. The theory is formed upon self-evident truths of consciousness that it exists, is structured, contains information, is irreducible to its components, and is exclusive to one unified experience [47]. From 
these axioms the following set of assumptions are formed: 1) A mechanism or set of mechanisms can only contribute to or be conscious only if it constrains the state of the system through allowing certain causes and effects; 2) A mechanism or set of mechanisms can only contribute to or be conscious only if its structure resulting in a certain cause and effect repertoire is irreducible to smaller parts without changing that repertoire; 3 ) Of an overlapping set of mechanisms, only one set can be conscious, and this is the one that is maximally irreducible to individual components resulting in the only consciousness existing in that system [47].

IIT claims that consciousness is not an all or non-property, but increases or decreases in proportion to the size of a system's repertoire of possible states. Derived from this claim, a central proposal of IIT is that the quality and quantity of an experience is specified by a maximally irreducible conceptual complex or structure that integrates large amounts of information [47]. This integration of information is generated by a mechanism above the information generated from its individual parts. IIT can be considered a modern form of panpsychism, the idea that consciousness is a universal feature or potential quality of all physical things. It follows this mystical philosophy due to its assertion that any maximally irreducible physical system performing information integration becomes conscious. IIT is brain-based due to its recognition that the corticothalamic system generates consciousness.

\subsection{The Dynamic Core Hypothesis}

The Dynamic Core Hypothesis (DCH) proposed by Edelman and Tononi is similar to IIT in that it focuses on complexity and informational aspects in producing consciousness, and it is the basis upon which IIT was formed [49]. DCH describes consciousness as a biological phenomenon and a product of evolution and development, completely explainable by neural activity [2], thus being a brain-based theory. It is similar to many other metastable theories in that it involves the global brain and corticothalamic activity [50]. The basis of DCH is the theory of Neural Darwinism, or neuronal group selection, in which neuroplasticity and long-range parallel connections among brain areas provide dynamic coordination among neuronal circuits necessary for consciousness [2]. Neural Darwinism proposes that connectivity in the brain occurs via epigenetic processes during development, producing structural diversity [51]. Postnatal environmental experiences then plastically modify the neural structure to be suited for real-world applications [51]. It describes a primary consciousness which exists simply in the "remembered present", lacking self-awareness. It also describes a higher-order consciousness which exists in humans allowing us to be conscious of being conscious [52].

The DCH's main assertion is that neuronal groups contribute directly to conscious experience only through bi-directional synchronistic interactions within the corticothalamic system that achieves high information integration in a short time [2]. For these neuronal groups to contribute to the sustainment of con- 
scious experience, they must additionally maintain state differentiation, a high level of complexity, and functionally interact with other widely distributed neuronal groups that create a cluster of interactivity [53]. Gamma and theta band synchrony is proposed to bind the dispersed neuronal groups into a seamless whole, and this reentrant synchrony defines connectivity within the core [2]. The dynamic core itself is not technically required to exist in any neural correlate, but is a process of neural interaction that can change in composition over time [53]. However, this process in newer versions of the model has been constricted to mainly consist of the ongoing reciprocal signaling within the cortex and between the cortex and thalamus, structured by the vast corticortical, corticothalamic, and thalmocortical connections throughout the brain [54].

The dynamic core allows for the synchronous patterns of metastable activity that connect and integrate the various functions of different brain areas, generating high levels of neural complexity [2]. The unified experience of many various qualia at any given moment is a complex of multiple sensory discriminations integrated within the dynamic core [2]. From this perspective, consciousness is a stream of unified mental formations that generate from the material structure that is the dynamic core. The boundaries of the dynamic core are themselves dynamic with neural clusters leaving and joining depending on influences from internal and external signals [54]. DCH describes how selection for the contents of the core is constrained by attentional, emotional, and reward signals originating from sub-cortical structures such as the brain stem [2]. Evidence for the $\mathrm{DCH}$ has been proposed by studies detecting electromagnetic signaling within the brain. In the study lead by Strinivasan observing binocular rivalry [55], conscious perception of a stimulus was associated with coherence among distinct and distant neuronal groups in the cortex.

\section{Critique and Comparison}

We draw comparisons to metastable consciousness models and hypotheses to show how the current scientific consensus on the topic is lacking a full view of the subject and why the DST is the predominant metastable theory (Table 1). We assert that the DST is the first all-encompassing and structurally sound model of consciousness due to its combined metastable and embodied cognition approach. Many theories focus on the neural correlates of consciousness. This is largely helpful, but it does not explain why certain neural areas are associated with consciousness. We assert that to understand consciousness, research must be conducted on the whole body and mind. According to the DST, certain neural correlates serve specific functions to consciousness, but the of ontology consciousness lies within the harmonic orchestra of membrane potential oscillations throughout the entire body and brain [29].

\subsection{Operational Architectonics Theory}

OAT is one of the few "world simulation" models that compare to the DST in 
Table 1. Comparison of consciousness models.

\begin{tabular}{|c|c|c|c|c|c|}
\hline Questions addressed by consciousness models: & $\begin{array}{l}\text { Integrated } \\
\text { Information } \\
\text { Theory }\end{array}$ & $\begin{array}{l}\text { Operational } \\
\text { Architectonics } \\
\text { Theory }\end{array}$ & $\begin{array}{l}\text { Dynamic Core } \\
\text { Model }\end{array}$ & $\begin{array}{l}\text { Global } \\
\text { Workspace } \\
\text { Theory }\end{array}$ & $\begin{array}{l}\text { 3D Default } \\
\text { Space Theory }\end{array}$ \\
\hline $\begin{array}{l}\text { Is it a global theory involving brain and body instead of a } \\
\text { brain-based theory? }\end{array}$ & No & No & No & No & Yes \\
\hline $\begin{array}{l}\text { Does the model include an internal, "virtual" 3D matrix } \\
\text { as the architectural foundation of consciousness? }\end{array}$ & No & Yes & No & No & Yes \\
\hline Does corticothalamic activity play a major role? & Yes & No & Yes & Yes & Yes \\
\hline Does it address underlying physiology? & Partially & Partially & Partially & Partially & Yes \\
\hline $\begin{array}{l}\text { Is it an expandable model? (can it be integrated with oth- } \\
\text { er models?) }\end{array}$ & Limited & Limited & Limited & Limited & Yes \\
\hline Does it address cardiorespiratory coherence? & No & No & No & No & Yes \\
\hline $\begin{array}{l}\text { Does it address the role of homeostasis and membrane } \\
\text { potential changes? }\end{array}$ & No & Partially & Partially & No & Yes \\
\hline $\begin{array}{l}\text { Is the thalamus a central hub for consciousness in the } \\
\text { model? }\end{array}$ & Partially & No & Partially & Partially & Yes \\
\hline Does the model explain the self? & No & Partially & No & No & Partially \\
\hline $\begin{array}{l}\text { Does the model explain how of visual and other sensory } \\
\text { inputs are integrated into a whole? }\end{array}$ & Partially & Partially & Partially & No & Yes \\
\hline \multicolumn{6}{|l|}{$\begin{array}{l}\text { Can the model illustrate the etiological deficits of the } \\
\text { following disorders: }\end{array}$} \\
\hline 1) contralateral neglect syndrome & No & Yes & No & No & Yes \\
\hline 2) phantom limb & No & No & No & No & Yes \\
\hline 3) motion sickness & No & Partially & No & No & Yes \\
\hline 4) vertigo & No & No & No & No & Yes \\
\hline 5) claustrophobia & No & No & No & No & Yes \\
\hline 6) Seasonal affective disorder & No & No & No & No & Yes \\
\hline 7) Central Pain syndrome & No & No & No & No & Yes \\
\hline \multicolumn{6}{|l|}{$\begin{array}{l}\text { Can the model explain the nature of the following } \\
\text { observations and self-experimentations: }\end{array}$} \\
\hline 1) Out of body experiences & No & Partially & No & No & Yes \\
\hline 2) Rubber Hand Experiment & No & No & No & No & Yes \\
\hline 3) Meditation Experiences & Partially & Partially & No & No & Yes \\
\hline $\begin{array}{l}\text { Can the model explain embodied cognition observations } \\
\text { by addressing underlying physiology on modulation of } \\
\text { the mind by the body? }\end{array}$ & No & No & No & No & Yes \\
\hline $\begin{array}{l}\text { Can the model explain changes in awareness during me- } \\
\text { ditation? }\end{array}$ & No & Partially & No & No & Yes \\
\hline $\begin{array}{l}\text { Does the model address how emotions and sensory info } \\
\text { are integrated? }\end{array}$ & Partially & Partially & Partially & Partially & Yes \\
\hline Does the model have experimental evidence? & No & Partially & Partially & Partially & Partially \\
\hline $\begin{array}{l}\text { Does the model include the autonomic nervous system } \\
\text { and its modulation? }\end{array}$ & No & No & No & No & Yes \\
\hline $\begin{array}{l}\text { Does the model correlate cellular events with global } \\
\text { consciousness events? }\end{array}$ & No & No & Partially & No & Yes \\
\hline $\begin{array}{l}\text { Does it detail the link between neural oscillations and } \\
\text { visual and auditory consciousness? }\end{array}$ & No & Yes & Yes & No & Yes \\
\hline
\end{tabular}


identifying the phenomenal, foundational architecture of consciousness as a subconscious 3D matrix into which all perceptions are placed [11] [24]. Both theories identify a functional isomorphism between the phenomena of this $3 \mathrm{D}$ space-time of consciousness and the space-time of macro bioelectric synchronizations among brain areas, and among the entire body according to DST. The existence of this subconscious 3D space and its essential nature are illustrated in the neurological condition of contralateral neglect syndrome in which spatial processing brain regions become damaged leading neglect and complete absence of consciousness regarding a particular side of one's perspective (Figure 2) [56] [57]. These patients are not aware of any "missing" or empty space, and will even act as if this space does not exist, even eating from only one side of their plate and dressing one side of their bodies [58]. Sensory stimuli from the "missing"
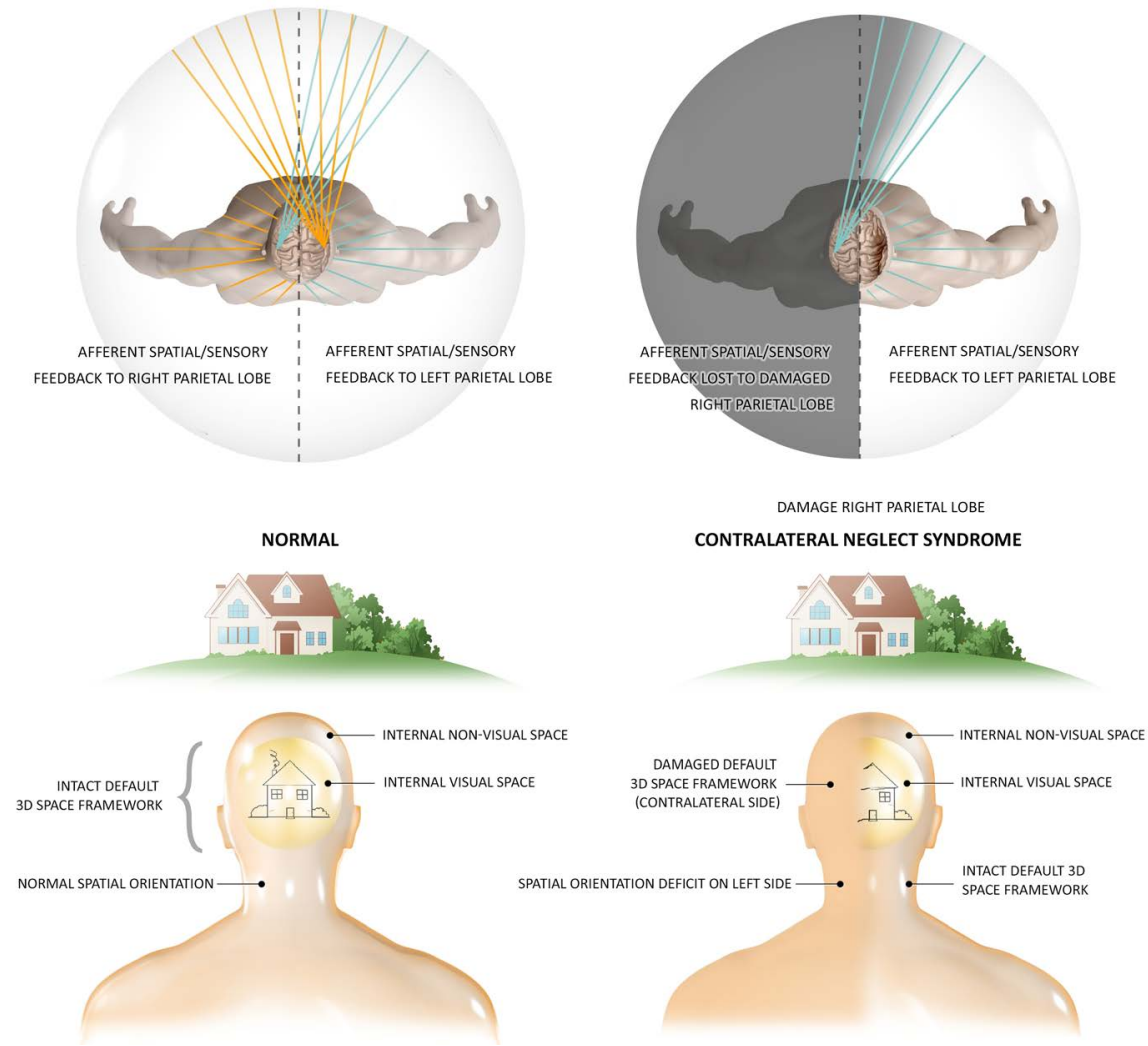

Figure 2. The subconscious structure of consciousness and contralateral neglect syndrome. Contralateral neglect syndrome results from damage to the right parietal lobe which maps spatial information on perceptual data regarding both sides of one's perspective. The left parietal lobe however maps information from only the right side of the perspective. Therefore, in those with contralateral neglect syndrome, perceptions intended to be mapped to the left side have no functional cortex to spatially orient them even though perceptual data is fed to said damaged cortex. These patients not only lack consciousness of such sensory stimuli, but the entire left side of space ceases to consciously exist. This is illustrated in the image above by the absence of the left side of bodily and visual perception in those with a damaged spatial framework (Figure by Lynsey Ekema, MSMI. Previously Published in [21]. Permission obtained by Creative Commons). 
side can still be fully processed, but however remain unconscious [56].

The DST and OAT identify significant psychological evidence (in addition to contralateral neglect syndrome) that this $3 \mathrm{D}$ matrix is the perceptual foundation for the "virtual space" of consciousness in that all perceptions are filled into this space [11] with an emphasis by DST on the top-down domination of this filling [59]. Thus, the "world simulation" is heavily influenced by cognitive predictions derived from memories and executive expectations. These evidences include dream studies which show that dreams are also organized within a spatially extended "virtual reality" in which the dreamer is spatially centered with a first-person perspective [60] [61]. The same can be observed in memory recollection and out-of-body experiences [11] [62]. OAT shares with DST the utilization of a metaphor of a global, harmonious, musical symphony to illustrate the cooperative oscillatory synchronization of distinct neuronal assemblies which bind conscious percepts [34]. Both theories identify significant research support for this assertion of global, multisensory integration in space and time not only in our unified conscious experience, but in the synchronization and integration of the respective neural modalities [63] [64] [65]. The DST takes this view further by asserting that the sensory organs throughout the body are also bioelectrically integrated into this global coherence [31].

A significant limitation of OAT is that it lacks the identification of a physiological mechanism for reentrant communication among dispersed neuronal modules that the DST [24], other metastable models [2] [46], and a large compendium of academic literature demonstrate the thalmocortical system is responsible for [66]-[71]. OAT provides very insightful and thorough detail of the operations of neuronal synchrony which surpasses the level the DST expounds. Although OAT lacks the communicative backbone of the thalamocortical system, its descriptions of metastable synchronization among distal, functional neural assemblies (OMs) provide mechanisms for cognitive operations such as multisensory perception that the DST explains only generally. By integrating the physiological detail of the DST and the operational detail of OAT, a greater comprehension of bioelectric operations leading to mind can be achieved.

The default mode network (DMN) is a self-referential network that holds a great importance to both the DST and OAT. Today's views on the DMN tie it to self-generated thought [72], social cognition [73], autobiographical memory, simulating the future [72], mind wandering, daydreaming, introspection, internal narrative [74], and the sense of self [75]. This brain network consists of low frequency oscillations between certain cerebral areas including the posterior cingulate cortex, medial prefrontal cortex, and the angular gyrus [72]. OAT has shown the DMN is comprised of consistently stable OMs (synchronistic bioelectric patterns and neural space-time) [76]. OAT ties this network to the first person sense of self and agency centered in the subconscious 3D matrix [11] [77]. The DST takes this further by asserting the DMNs central role in the foundation of this 3D matrix [24]. The DST ties in bodily electrical oscillations into this 
network as part of its body-mind framework by citing influence on the DMN by cardio and respiratory waves [78]. Furthermore, the DST propounds that the low-amplitude oscillations of the DMN in association with cardiorespiratory oscillations create a framework upon which the faster oscillations of consciousness can be built [30].

The DST and OAT both link DMN physiology with the self, meditation, and associated experiences. Work by the Fingelkurts and other researchers has identified the DMN as a preferable neural correlate for the sense of self [79] [80]. The Fingelkurts identified in their research a three-part modularization of the DMN which showed altered activity relative to each three subnet modules after extensive mediation training [80]. They describe how their research indicates each module contributes specific functions in forming a complex self-hood [80]. Both the DST and OAT describe the self being physically centered in the externalized perceptual reality of which the foundation is the subconscious 3D matrix [24] [80]. The DST further describes this center as being the physical location of the thalamus as all perceptual streams are coordinated by and around it [24].

Research and accounts from experienced mediators demonstrates how long-term meditation leads to increased integrity of frontal DMN connectivity which is shown to be responsible for this sense of "center" and first-person perspective [73] [80] [81] [82]. Experienced meditators report a sharper sense of a "self-center" [83] while simultaneously experiencing loss of self-boundaries and physical embodiment [84] [85]. This disembodiment and loss of self-boundaries is asserted by the Fingelkurts with research support to be due to decreased functional connectivity among the posterior subnets of the DMN [80]. The DST asserts this experience is due to an expansion of awareness of the normally subconscious 3D matrix underlying all experience and the accurate identification of this infinite 3D virtual space with one's own being [86]. The DST advances the concepts of self and the DMN through linking it to respiratory and cardio activity, helping to explain some effects of deep-breathing meditative practices [30]. The DST asserts that the increased functional connectivity seen in the frontal subnet of the DMN could be caused by rhythmic respiration generated inhibitory impulses and cardio-respiratory phase synchronization [86].

\subsection{Global Workspace Theory}

GWT consists of multiple detailed hypotheses aimed at understanding the differences between conscious and unconscious brain events [46], disregarding the possibility of consciousness involving bodily events. The DST also aims to understand this difference, instead associating consciousness with higher frequency oscillations and integrated and complex electrophysiological activity which require the thalamus. According to GWT, the contents of experience are associated with temporary "broadcasts" of reentrant neural activity, specifically in the thalamocortical network, considering it a vast interactive signaling medium [46]. Strong similarities lie between GWT and the DST in the shared importance 
of globally distributed, synchronous activity across the thalamocortical network, as well as its parallel processing capabilities [24] [46]. The DST completes this view by further asserting the importance of oscillatory harmony across the entire body, not just the central nervous system [24]. GWT fails to include explanations on aspects of emotion and the top-down dominance of the sensory system. GWT seems to ignore baseline, resting, or "silent" consciousness by describing consciousness as highly activated neural responses to stimuli. The DST explains how a baseline consciousness is always active (in an awake person) via the DMN and lower frequency bioelectric oscillations which maintain the internal 3D space which allows for experiential awareness [30]. GWT theory misidentifies the vast role unconscious physiology plays in the structure of consciousness by separating it from globally active networks which are propounded to be conscious. The DST however explains how all bioelectric cellular activity is part of a global network, citing the evidence that lower frequency oscillations it associates with the unconscious support, underlie, organize, and modulate higher frequency ones it associates with consciousness [87] [88].

The DST shares the functional hub view of the corticothalamic system underlying consciousness with GWT [24], however GWT proposes a dynamic 'global workspace' hub of activity binding and propagation consisting of varying anatomical components of the corticothalamic network which are themselves small world network hubs [46], while the DST focuses on the thalamus as a central anatomical hub which integrates cortical and sensory receptor activity within a virtual 3D matrix, creating a unified experience [21]. Both models hold the assertion that the contents of experience are associated with the global networking of the thalamocortical system [24] [46]. The perspective of GWT is that conscious contents may emerge anywhere in this corticothalamic network [46] (Figure 3), however, the DST explains how parietal regions of this network are required for consciousness because they spatially map percepts [89]. While GWT does acknowledge this spatial quality, it limits it to visual perception [46]. Both models stress the bidirectionality, or reentrant neural connections in the corticothalamic network that bind and broadcast information throughout the brain [24] [46]. Evidence has supported both model's metastable assertions that although each separate brain area processes distinct features of the overall integrated experience, synchrony between neural modules binds these features together into a seamless whole [64]. The main difference between these two model's metastable assertions is that a global oscillatory framework has been proposed for synchronization that spans every cell of the body, creating a truly unified, bioelectric framework [30]. This global framework creates an unconscious basis upon which consciousness is built [30].

The corticothalamic perspective of consciousness has been based on a variety of scientific findings including brain imaging studies, intracranial stimulation recordings, and empirical analysis of conscious and unconscious brain conditions [38] [46] [66]. While GWT acknowledges the neural oscillations as highly 


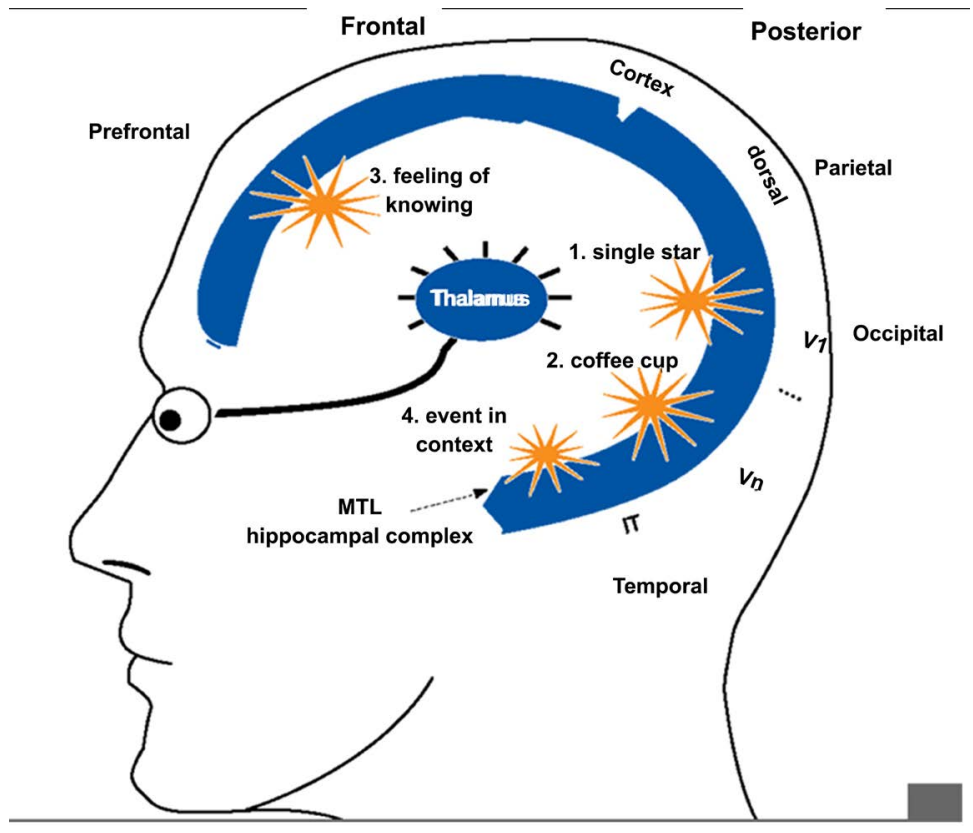

Figure 3. Consciousness Can Occur Anywhere in the Corticothalamic loop. This figure illustrates the GWT concept that consciousness can arise from anywhere in the corticothalamic network, depending on which activity is bound and broadcast to the rest of the network. Each yellow starburst indicates a site of binding and global broadcasting leading to consciousness. Only one of these sites can be the any-to-many "activity winner" at any given time as global broadcasts interfere. Activity dominating from certain brain areas leads to unique experiences such as the experience of a single star broadcasting from $\mathrm{V} 1$ in the occipital lobe. (Previously published in [46]. Permission obtained from Creative Commons License).

functional, it however undervalues the oscillatory nature of the corticothalamic system, instead preferring a perspective of "adaptive resonance" among reentrant corticothalamic pathways [46]. Our perspective is that this bioelectric oscillatory nature is essential to mass synchronization of vast cortical/sensory modules and in the physical existence of consciousness [30] by creating an electromagnetic architecture which GWT leaves out of its global workspace [90]. Baars et al. indicate that through the corticothalamic system, the cortex "mostly talks to itself" [46]. However, the synchronization of the sensory organs with their cortical counterparts with the cortex talking more to the sensory organs than the sensory organs to the cortex has been stressed by the DST [31]. A strong benefit to maintaining perceptual representations in a physical form at the site of the sensory organs frees up the need for the brain to maintain internal, mental sensory representations.

A criticism of the GWT lies in the nature of unconscious and conscious neural activity. Suggested by GTW, significant activation of concepts in the brain leads to consciousness of those concepts [91]. However, it is clear that generality of concepts transcend the experiences they apply to [91]. For instance, take the concept of a chair. If you try to visualize the concept of "chairness", it simply cannot be done. Whenever the concept of a chair is attempted in visualization, 
only certain tokens of chairs from certain vantage points can be experienced. Categories cannot be perceived. It is clear that abstractions that can be understood and processed by the unconscious mind cannot be consciously experienced. For instance, consider the word culture. When we think of this we understand its meaning and implication but cannot consciously experience any particular concept of culture although we can identify it. This implies that strong activation of neural concepts is not sufficient for entrance into consciousness. This implication strengthens our perspective that consciousness is built upon a foundation of "world simulation" that is not necessarily based on quantity of activation and global access of brain activity.

By identifying an underlying, global, bioelectric structure creating an unconscious $3 \mathrm{D}$ virtual matrix upon which a conscious world simulation can be built, the DST has advantages over GWT in explaining the ontology of consciousness. The DST, through its world simulation approach, accounts for criticisms of the GWT such as how the existence of global neural broadcasting is not sufficient for consciousness of certain concepts such as the lack of ability for people to qualitatively perceive categories, stressing the necessity of a spatial component to all qualia. Through an embodied cognitive approach in stressing the role sensory receptors play in consciousness, the DST helps explain the strong influence the body has over the cognitive mind, providing a means for voluntarily modulating mood, stress, and cognition. GWT describes a functional role of consciousness, but doesn't seem to make much progress towards solving the "hard problem of consciousness". By describing an electromagnetic field cognitive architecture, the DST provides a physical substrate for consciousness, thereby providing a starting point for solving this hard problem. While the global workspace architecture surely provides an efficient means of processing information, it doesn't provide an explanation as to why experience needs to be an aspect of global availability and access. GWT explains that top-down attention helps determine which information is task-relevant and enters global availability. We criticize the lack of specification of how attention "decides" which information is relevant.

\subsection{Integration Information Theory}

IIT attempts to explain differences between states varying in levels of consciousness such as the awake/asleep state as well as the differences in brain areas involved in generating consciousness contrasted with those that don't such as the cortex versus the cerebellum [92]. The DST attempts to do the same focused on a similar concept of metastable information integration, however, it includes the thalamus and the sensory receptors in a global manner. Both the DST and IIT explain how conscious arises from processes that arise from a system that cannot be reduced to the sum of its parts; in the case of the DST, this system is the whole body and brain. The inclusion by the DST of the thalamus as the ultimate integrator of information is a substantial difference between the two theories. IIT propounds that consciousness is minimized or eliminated during slow-wave 
sleep because the irreducible system leading to consciousness becomes significantly smaller and reconfigured, and the neural networks are much less complex and more disconnected due to bistability [92] [93]. The DST on the other hand describes how the disconnect between the thalamus and cortex during slow-wave sleep [94] leads to unconsciousness because the thalamus ceases to integrate and unify the information from the body and corticothalamic network into a metastable harmony that generates the $3 \mathrm{D}$ world simulation of consciousness. IIT asserts that the cerebellum is not part of consciousness because it is a feedforward network that has multiple independent zones which do not form a large complex, therefore not form a large system that integrates information [92]. The DST asserts that the cerebellum is not significantly involved in the production of consciousness because it does not influence the generation of the world simulation, only the motor interaction with it. It could be understood that destruction of the cerebellum alters consciousness in that it disrupts the conscious perception of coordination between the body and physical environment.

We propound that IIT has gained a strong acceptance and popularity by playing on what we know to be true about the brain being a vast spanning web of integrated information. Many people have an intuition about consciousness being an integrated whole resulting from the full spectrum of brain activity, however, most networks in the brain are actually unconscious [95], and unconscious activity precedes the arrival of consciousness [96]. In describing consciousness as resulting from information processing systems, IIT fails to account for why the majority of cognitive activity is unconscious. The DST however posits this unconscious activity as the foundation of consciousness [30]. IIT is one of the few theories that incorporates mathematics, however, an over focus on this mathematical aspect may take attention away from the empirical hurdles that must be overcome to develop an accurate biological model. IIT provides important insight into the nature of informational integration and consciousness; however, there are empirical observations that seem to disprove IIT, which the DST does explain. The terminologies used in the IIT model are often esoteric and baffling to those who try to understand it on the first exposure. While this may be necessary, it brings forth the criticism that IIT is trying to persuade its audience of its accuracy by perplexing them.

The axioms of conscious proposed by IIT are certainly insightful and verifiable, however they seem to lack support for the theories own fundamental concepts. In the principle of exclusion provided by IIT, only one consciousness can be maintained by any system [47]. Even if its components satisfy the other requirements for consciousness, that consciousness would be suppressed by the greater whole. This however leads to the conclusion that if a group discussion leads to large information integration, sufficient for consciousness, then the individual members of that group would cease to be conscious. Similar to GWT, IIT assumes that concepts such as the "chairness" described, can also reach conscious awareness due to these concepts being involved in the overall informa- 
tional system of the brain. While it describes the spatially structured nature of consciousness through its composition axiom [47], IIT lacks the important explanation of the "world simulation" function of consciousness that the DST [21] and other authors [97] [98] [99] [100] [101] describe.

The DST describes how during meditation, some can achieve an awareness of this unconscious spatial matrix, leading to experiences of identifying with and experiencing a vast, boundary-less, empty space [102]. Unlike GWT, IIT identifies a baseline, silent, or naked state of experience which can be reached via meditation [103] due to silent but functionally connected neurons which while are not actively transmitting information, are part of a complex with positive $\Phi$ [92]. The DST doesn't require silent neurons or mediation for naked awareness, including it as a foundation of consciousness which is always idle in the awake state powered by the DMN and baseline cardiorespiratory oscillations [30]. This naked space is filled in with oscillations giving rise to qualia that arise from corticothalamic feedback loops which are integrated by the thalamus [24]. IIT does not identify a functional importance to these lower frequency baseline oscillations which the DST proposes are increased during meditation due to increased cardio-respiratory synchronization, thus leading to dynamic changes in awareness [86]. By describing how body rhythms affect neural oscillations via an embodied cognitive approach, the DST can explain mechanisms of therapeutic benefits of techniques such as meditation.

The axioms of consciousness that Tononi proposes as the basis of IIT are explained by the fundamentals of the DST. Tononi asserts that every experience is structured with different aspects into a singular integrated whole [47]. The DST shares this assertion and furthers its notion by describing how this integration occurs in the human mind and into what type of structure the qualia is integrated (a virtual 3D matrix). IIT assumes this type of experience occurs with any sufficient information integrating system and in a strong similarity to the DST and other models, describes the corticothalamic system as the main neural substrate for integrating vast neural information [1]. The DST is founded on the structure of consciousness occurring as a $3 \mathrm{D}$ space just as in the $3 \mathrm{D}$ world in which we live. Tononi seems to apply the familiar structure of human consciousness to any potentially conscious physical system whereas the DST proposes distinct cortical and thalamic systems (i.e. parietal lobe) are necessary and partially responsible for such a structure without which human consciousness could not exist [89].

The necessity of the parietal lobe in consciousness is due to its role in constructing the spatial dimension of the internal replication of external space [89] [104] [105]. The spatial dimension of sensation is necessary for all experience to occur and this has been exemplified through an understanding of contralateral neglect syndrome as we have explained. This syndrome indicates that if sensation can't be given a spatial characteristic by the parietal lobe, it cannot enter into conscious experience. According to his composition axiom, Tononi asserts 
that this experiential spatial characteristic is a fundamental result of any irreducible information processing and integrating system, however, when the parietal lobe is damaged leading to contralateral neglect, the brain still fully processes stimuli from the missing side unconsciously and the patient can maintain blindsight of these stimuli [56]. Unconscious stimuli from the neglected field of these patients significantly increased response times to a subsequent word related to the neglected stimuli, such as bat-ball, tree-apple, compared to unrelated words like bed-apple [106], indicating they are part of the irreducible system and thus should be conscious. If Tononi is correct in stating the spatial characteristic is a result of any irreducible system, then contralateral neglect syndrome would not exist. The same effect can be seen in the phenomenon of cortical blindsight in those with damage to the visual cortices. Although they can to some degree detect, localize, discriminate, and respond to visual stimuli in restricted ways, they cannot consciously experience the stimuli or understand why they are able to respond to it [107] [108]. There is so much complex information integration that happens out of conscious awareness, that it seems the only way the assertions of IIT could be completely accurate is if there were multiple consciousnesses inside one brain. It could be assumed that if multiple non-overlapping irreducible networks somehow separate in the brain existed simultaneously in one brain, more than one consciousness could exist in that brain, and this is actually an assertion of IIT, even in the everyday life of healthy people [47].

IIT describes the sensory receptors as external inputs that enable consciousness through maintaining excitability in the brain, thus being a "background condition" to consciousness [92]. IIT asserts that sensory connections are feedforward, however if it would acknowledge that if indeed the sensory receptors are bioelectrically synchronized with their respective cortical sites and thus contain feedback as proposed by the DST described earlier in this article, then these sensory receptors would be part of the irreducible information integrating system leading to consciousness. They would simultaneously be a part of cause and effect repertoire according to the view of the DST that they cause certain afferent stimuli and receive efferent effects by the cortex. Thus, according to this concept, the sensory receptors are in a sense part of the corticothalamic system. This is due to the feedback mechanisms proposed by DST in which information is fed back to the sensory receptors. According to IIT, feedforward networks are not conscious as they lack any information integration [92]. DST accounts for this in its account of feedback to the sensory receptors and the feedback loops of the corticothalamic network which integrate information [24]. While IIT emphasizes the important role of these corticothalamic loops, it does not recognize the importance of feedback to the body nor the crucial role of the thalamus as a hub of conscious experience due to its integration of the integrating corticothalamic loops, thus performing a sort of "meta-integration".

We propose that information integration as described by Tononi is a neces- 
sary condition, but not a sufficient condition to consciousness. IIT comes to some strange, counterintuitive conclusions through describing consciousness as a system with high $\Phi$. Easily explainable, high $\Phi$ systems have been proposed that could exist as 2D logic grids on a compact disc which would have vast quantities of consciousness [92] [109]. This goes against common sense understandings however, especially since they would have an extremely greater quantity of consciousness than an average person. Tononi has conceded that this would be the case, stating that the commonsense intuition that such a system could not be conscious is based on pre-theoretical assumptions comparing it to once held assumptions that the earth is flat and the sun revolves around the earth [110]. Tononi mentions other counterintuitive propositions of IIT which include 1) A photodiode with memory is minimally conscious , 2) A functional cortex which is at a time silent with no neurons firing or oscillating would still be conscious, and 3) A functional 2D grid could be highly conscious even if silent, meaning all its gates are off [110]. By describing consciousness not only as integrated information, but as resulting from a bioelectric synchrony of information throughout the body and brain creating a world simulation via the thalamus, the DST accounts for these counterintuitive implications and explains the difference in conscious states such as awake/sleep and the difference in consciousness generation between areas such as the cortex and cerebellum through the inclusion of an anatomical central hub which unifies vast bioelectric, neural and bodily activity creating a world simulation within, thus describing a physical substrate, electromagnetism, as the physical source of consciousness.

\subsection{Dynamic Core Hypothesis}

Just like the DST, IIT, and GWT, the DCH places a strong importance on neuronal integration and synchronization via the corticothalamic system, describing it as a functional hub [50]. These metastable models suggest that global brain dynamics emerge from cooperative interactions between diverse neuronal groups, and that consciousness can only be explained by these distributed neural processes and not local properties of neurons [111]. The DST additionally asserts reentrant activity occurs between these neural groups as well as the sensory organs [31]. These models explain how different parts of the nervous system cooperate to produce a unified whole. Both the DST and DCH characterize the thalmocortical network interactions as reentrant, parallel, and recursive, with the DCH describing consciousness as emerging from anywhere is the corticothalamic or corticortical arena [2]. The DST includes a similar concept of corticothalamic integration to that of the dynamic core, however, further proposes a harmonious oscillatory unity not just between cortical areas and the thalamus, but across all cells of the body, requiring the thalamus for consciousness to emerge [24]. The DCH stresses the importance of reentrant activity in creating the functional connectedness of the core [2], however, the DST necessitates bioelectric oscillatory activity in creating consciousness which is reentrant but also 
creates the substrate of consciousness, electromagnetism. The substrate according to DCH would be information.

In contrast to the $\mathrm{DCH}$, the DST describes how information from the entire cortex is integrated by the thalamus into consciousness, not just certain components. While some areas may not be activated at any given time, they still are part of the neural network and contain information via baseline activity which is a ubiquitous property of the brain [112]. For instance, the part of your brain that processes color is not activated if there is only gray in your visual environment or imagination, however, that part of the brain still contributes to experience [113]. On the other hand, if that color brain region is damaged due to a lesion, then the patient would not see gray and would not see any color, lacking understanding that the visual scene is missing color [114]. We propose the contribution of non-stimulated neuronal assemblies is done by signifying the absence of color through its low frequency oscillatory baseline activity which plays a key role in perception and is an aspect of neural coding [112]. Thus, information from the entire cortex is integrated by the thalamus to create our unified experience which includes information encoding the lack of certain qualia which includes the understanding that the qualia lacks something.

The DCH stresses that at any given time certain clusters of neurons are part of the dynamic core while others are not, even if they are equally active and have been part of the core previously [111]. The DST also posits that different neuronal clusters are integrated into the default space and therefore into conscious awareness, however, all neuronal groups play a role in the development of the unconscious aspects of the default space [24]. A main difference as described previously via contralateral neglect and the parietal lobe, is that the default space requires those neuronal groups responsible for spatial processing to be intact for conscious experience to occur [89], while the $\mathrm{DCH}$ asserts a lack of requirement for any neuronal groups in consciousness [111]. The DCH does describe how emotions originating from sub-cortical regions may constrain the selection of which brain regions become part of the dynamic core [2]. The embodied cognitive approach of the DST however stresses the role of cardio-respiratory coherence in modulating emotional states in part via the autonomic nervous system [115]. Citing evidence that positive emotions are correlated with higher rates of synchronization and negative emotions with desychronization among body and brain rhythms [116] [117], the DST proposes a foundational oscillatory architecture of which the cardiorespiratory system is a major component. Through including the body in the cognitive structure generating consciousness, the DST extends metastable assertions and explains a basic mechanism for how voluntarily modulating one's body can allow them to modulate their minds.

The DCH, IIT, and DST all stress the unity of conscious experience and that this experience occurs from a particular point of view. The DST and OAT however provide the structure and frame of this perspective [11] [24]. Our model describes how this perspective is experienced in respect to the thalamus and de- 
tails how alterations in corticothalamic activity can lead to an altered perspective as experienced in out-of-body experiences [62]. The dynamic core does not refer a unique, invariant functional cluster of neurons, as the core may change in composition over time [50]. The DST does recognize that varying clusters of activity will have their own local synchronies, however, it posits a global synchrony orchestrated by the thalamus with an invariant idling system of the DMN and cardiorespiratory oscillations that maintain the default space at all waking times [30]. The DCH proposes that higher-order consciousness of humans arises when symbolic representations and language are incorporated into the core [118]. This includes abilities of abstraction allowing us to be conscious of being conscious. While both theories associate high frequency brain waves with consciousness [118], the DST stresses the importance of (sub/un)conscious, lower frequency activity in forming a foundation for conscious activity to be organized [30]. By associating pervasive lower frequency oscillations with unconsciousness [118] instead of a basis for conscious activity to build upon, the DCH has ignored the vast low frequency activity which occurs throughout the body and brain during awake states. There is significant support for the assertion that low frequency activity provides long-range communication [119], modulates higher frequency activity [87], and forms a foundation for higher frequencies to organize upon [88]. It is by this mechanism we propose the embodied cognitive perspective that respiration modulates mental activity, by globally entraining neural oscillations [120].

By including the radical idea that sensory receptors are part of the dynamic oscillatory complex as a component in the creation of a world simulation within, the DST provides insight into how this simulation is maintained and how it helps us quickly react to the external world [21]. By maintaining mental representations on the sensory receptors, expectations about incoming sensory stimuli derived from the state of the simulation can be integrated with the actual stimuli, priming the receptors to receive certain stimuli and to discard other stimuli. The next "frame" of the simulation can thereby by updated in near real time as the processing powers of cortex are brought to the sensory receptors allowing a continuous manipulation of the simulation [21]. The thalamus integrates the oscillatory modules for each sensory pathway into a higher level, global synchrony responsible for integrated experience. The DCH provides additional insight, which can be understood as a component of the DST. The fact that the DST is compatible with the theories reviewed and in a sense is an extension of them illustrates its comprehensive nature and power to explain the various empirical observations currently in the literature.

\section{Conclusions}

Currently popular metastable consciousness models offer many insights into cognition; however, they are incomplete and insufficient to truly describe biological mechanisms of consciousness. Through a comparison with these models, 
we have formed an assertion that the DST completes the cognitive architectural perspective on consciousness that other models have ignored or failed to identify by incorporating the entire body into the metastable architecture laid out by these models. By including the entire body, we have provided mechanisms for experimental observations of the bodily effects on cognition and mental states as well as explaining the benefits of mind-body techniques such as breathing exercises. By extending the "operational information integration" system to the sensory receptors, we have provided a means for mental representations to be stored in the highest topographical sense, on the sensory receptors themselves.

The DST consists of components of top models of consciousness such as metastability, phenomenological axioms, and the important role of the corticothalamic system in the creation of consciousness while extending these concepts with novel propositions. The DST puts forth the radical notion that consciousness arises not solely from the brain, but emerges from bioelectric operations occurring throughout the entire body. It identifies a bioelectric architecture which consists of harmonized and synchronized membrane potential oscillations occurring in every cell. The novelty of the DST is further seen in its description of the layers of such architecture and its role in creating an active simulation of the external world. By approaching the modeling of consciousness from a holistic instead of a reductionist view, the DST may further understanding of the mind-body connection.

The literature on embodied cognition lacks insight into consciousness, and via the DST, we have physiologically extended consciousness to the body. Through doing so, we have provided a means for a seamless experience of the world which occurs in near real time and provided a means for one to voluntarily modulate one's mental state, primarily by modulating breathing pattern. We have illustrated the similarities to other metastable brain-based models, their criticisms the DST accounts for, and how it encompasses the strengths of other models while expanding their scope. By showing the incompleteness and deficits of each of the models, we have shown how the DST fills in the blanks and amends the faults of other models, ultimately forming a profound and paramount model. It remains to be determined whether direct studies on this model will elucidate its intricacies and validate its concepts.

\section{Acknowledgements}

We would like to thank Dr. Vernon Barnes for editing the manuscript.

\section{Author Contributions}

Theory developed by RJ with some writing with majority of the manuscript written by CB.

\section{Conflicts of Interest}

The authors declare no conflict of interest. 


\section{Funding}

All funding provided by the Charitable Medical Healthcare Foundation

\section{References}

[1] Tononi, G. (2004) An Information Integration Theory of Consciousness. BMC Neuroscience, 5, 42. https://doi.org/10.1186/1471-2202-5-42

[2] Edelman, G.M., Gally, J.A. and Baars, B.J. (2011) Biology of Consciousness. Frontiers in Psychology, 2, 4. https://doi.org/10.3389/fpsyg.2011.00004

[3] Gweon, H. and Saxe, R. (2013) Developmental Cognitive Neuroscience of Theory of Mind. In: Rubenstein, J. and Rakic, P., Eds., Neural Circuit Development and Function in the Brain: Comprehensive Developmental Neuroscience, Elsevier, Amsterdam. https://doi.org/10.1016/B978-0-12-397267-5.00057-1

[4] Chalmers, D.J. (1995) Facing up to the Problem of Consciousness. Journal of Consciousness Studies, 2, 200-219.

[5] Perlovsky, L. and Kozma, R. (2007) Neurodynamics of Cognition and Consciousness. Springer, Berlin. https://doi.org/10.1007/978-3-540-73267-9

[6] Kelso, J.A.S. and Tognoli, K. (2007) Toward a Complementary Neuroscience: Metastable Coordination Dynamics of the Brain. In: Perlovsky, L. and Kozma, R., Eds., Neurodynamics of Cognition and Consciousness. Understanding Complex Systems, Springer, Berlin, 39-59. https://doi.org/10.1007/978-3-540-73267-9_3

[7] Fingelkurts, A.A. and Fingelkurts, A.A. (2004) Making Complexity Simpler: Multivariability and Metastability in the Brain. International Journal of Neuroscience, 114, 843-862. https://doi.org/10.1080/00207450490450046

[8] Steinmetz, P.N., et al. (2000) Attention Modulates Synchronized Neuronal Firing in Primate Somatosensory Cortex. Nature, 404, 187. https://doi.org/10.1038/35004588

[9] Peter, B. and Jon, F.M. (2001) Book Review: Cortical Network Resonance and Motor Activity in Humans. The Neuroscientist, 7, 518-526. https://doi.org/10.1177/107385840100700608

[10] Varela, F., et al. (2001) The Brainweb: Phase Synchronization and Large-Scale Integration. Nature Reviews Neuroscience, 2, 229. https://doi.org/10.1038/35067550

[11] Fingelkurts, A.A., Fingelkurts, A.A. and Neves, C.F.H. (2010) Natural World Physical, Brain Operational, and Mind Phenomenal Space-Time. Physics of Life Reviews, 7, 195-249. https://doi.org/10.1016/j.plrev.2010.04.001

[12] Sporns, O. and Kötter, R. (2004) Motifs in Brain Networks. PLOS Biology, 2, e369. https://doi.org/10.1371/journal.pbio.0020369

[13] Wilson, R.A. and Foglia, L. (2017) Embodied Cognition. In: E. N.Z., Ed., The Stanford Encyclopedia of Philosophy, Metaphysics Research Lab, Stanford University, Stanford.

[14] Panksepp, J. (2012) In Defence of Multiple Core Affects. In: Zachar, P. and Ellis, R.D., Eds., Categorial Versus Dimensional Models of Affect: A Seminar on the Theories of Panksepp and Russell, John Benjamins Publishing Company, Amsterdam, 31-78. https://doi.org/10.1075/ceb.7.02pan

[15] Kiverstein, J. and Miller, M. (2015) The Embodied Brain: Towards a Radical Embodied Cognitive Neuroscience. Frontiers in Human Neuroscience, 9, 237. https://doi.org/10.3389/fnhum.2015.00237

[16] Piccinini, G. and Bahar, S. (2013) Neural Computation and the Computational Theory of Cognition. Cognitive Science, 34, 453-488. 
https://doi.org/10.1111/cogs.12012

[17] Wilson, A.D. and Golonka, S. (2013) Embodied Cognition Is Not What You Think It Is. Frontiers in Psychology, 4, 58. https://doi.org/10.3389/fpsyg.2013.00058

[18] Favela, L.H. (2014) Radical Embodied Cognitive Neuroscience: Addressing "Grand Challenges" of the Mind Sciences. Frontiers in Human Neuroscience, 8, 796. https://doi.org/10.3389/fnhum.2014.00796

[19] Ionescu, T. and Vasc, D. (2014) Embodied Cognition: Challenges for Psychology and Education. Procedia-Social and Behavioral Sciences, 128, 275-280. https://doi.org/10.1016/j.sbspro.2014.03.156

[20] Neuhoff, C.C. and Schaefer, C. (2002) Effects of Laughing, Smiling, and Howling on Mood. Psychological Reports, 91, 1079-1080.

[21] Jerath, R. and Beveridge, C. (2018) Top Mysteries of the Mind: Insights from the Default Space Model of Consciousness. Frontiers in Human Neuroscience, 12, 162. https://doi.org/10.3389/fnhum.2018.00162

[22] Searle, J.R. (1992) The Rediscovery of the Mind. MIT Press, Cambridge.

[23] Freeman, W.J. (1992) Tutorial on Neurobiology: From Single Neurons to Brain Chaos. International Journal of Bifurcation and Chaos in Applied Sciences and Engineering, 2, 451-482. https://doi.org/10.1142/S0218127492000653

[24] Jerath, R., Crawford, M.W. and Barnes, V.A. (2015) A Unified 3D Default Space Consciousness Model Combining Neurological and Physiological Processes That Underlie Conscious Experience. Frontiers in Psychology, 6, 1-26.

[25] Jerath, R.C., Barnes, V.A. and Jensen, M. (2018) Micro-Calibration of Space and Motion by Photoreceptors Synchronized in Parallel with Cortical Oscillations: A Unified Theory of Visual Perception. Medical Hypotheses, 110, 71-75. https://doi.org/10.1016/j.mehy.2017.11.005

[26] Min, B.K. (2010) A Thalamic Reticular Networking Model of Consciousness. Theoretical Biology and Medical Modelling, 7, 10. https://doi.org/10.1186/1742-4682-7-10

[27] Cudeiro, J. and Sillito, A.M. (2006) Looking Back: Corticothalamic Feedback and Early Visual Processing. Trends in Neurosciences, 29, 298-306. https://doi.org/10.1016/j.tins.2006.05.002

[28] Ward, L.M. (2013) The Thalamus: Gateway to the Mind. Wiley Interdisciplinary Reviews. Cognitive Science, 4, 609-622. https://doi.org/10.1002/wcs.1256

[29] Jerath, R., et al. (2017) The Dynamic Role of Breathing and Cellular Membrane Potentials in the Experience of Consciousness. World Journal of Neuroscience, 7, 66-81. https://doi.org/10.4236/wjns.2017.71007

[30] Jerath, R. and Crawford, M.W. (2015) Layers of Human Brain Activity: A Functional Model Based on the Default Mode Network and Slow Oscillations. Frontiers in Human Neuroscience, 9, 248. https://doi.org/10.3389/fnhum.2015.00248

[31] Jerath, R., et al. (2016) How Lateral Inhibition and Fast Retinogeniculo-Cortical Oscillations Create Vision: A New Hypothesis. Medical Hypotheses, 96, 20-29. https://doi.org/10.1016/j.mehy.2016.09.015

[32] Jerath, R., et al. (2017) Sensory Consciousness Is Experienced through Amplification of Sensory Stimuli via Lateral Inhibition. World Journal of Neuroscience, 7, 244-256. https://doi.org/10.4236/wjns.2017.73020

[33] Seth, A.K. (2016) The Real Problem.

[34] Fingelkurts, A.A. and Fingelkurts, A.A. (2001) Operational Architectonics of the 
Human Brain Biopotential Field: Towards Solving the Mind-Brain Problem. Brain and Mind, 2, 261-296. https://doi.org/10.1023/A:1014427822738

[35] Fingelkurts, A.A. and Fingelkurts, A.A. (2005) Mapping of the Brain Operational Architectonics. In: Chen, F.J., Ed., Focus on Brain Mapping Research, Nova Science Publishers Inc., New York, 59-98.

[36] Fingelkurts, A.A., Fingelkurts, A.A. and Neves, C.F.H. (2009) Phenomenological Architecture of a Mind and Operational Architectonics of the Brain: The Unified Metastable Continuum. New Mathematics and Natural Computation, 5, 221-244. https://doi.org/10.1142/S1793005709001258

[37] Singer, W. (2001) Consciousness and the Binding Problem. Annals of the New York Academy of Sciences, 929, 123-146. https://doi.org/10.1111/j.1749-6632.2001.tb05712.x

[38] Freeman, W.J. (2007) Indirect Biological Measures of Consciousness from Field Studies of Brains as Dynamical Systems. Neural Networks, 20, 1021-1031. https://doi.org/10.1016/j.neunet.2007.09.004

[39] Fingelkurts, A.A. and Fingelkurts, A.A. (2014) Present Moment, Past, and Future: Mental Kaleidoscope. Frontiers in Psychology, 5, 221-244. https://doi.org/10.3389/fpsyg.2014.00395

[40] Shanahan, M. and Baars, B. (2005) Applying Global Workspace Theory to the Frame Problem. Cognition, 98, 157-176. https://doi.org/10.1016/j.cognition.2004.11.007

[41] TechNet (2012) Blackboard Design Pattern.

[42] Baars, B.J. (1997) In the Theatre of Consciousness: Global Workspace Theory, a Rigorous Scientific Theory of Consciousness. Journal of Consciousness Studies, 4, 292-309.

[43] Shanahan, M. (2006) A Cognitive Architecture That Combines Internal Simulation with a Global Workspace. Consciousness and Cognition, 15, 433-449. https://doi.org/10.1016/j.concog.2005.11.005

[44] Baars, B.J. (2002) The Conscious Access Hypothesis: Origins and Recent Evidence. Trends in Cognitive Sciences, 6, 47-52. https://doi.org/10.1016/S1364-6613(00)01819-2

[45] Baars, B.J. (2005) Global Workspace Theory of Consciousness: Toward a Cognitive Neuroscience of Human Experience. Progress in Brain Research, 150, 45-53. https://doi.org/10.1016/S0079-6123(05)50004-9

[46] Baars, B.J., Franklin, S. and Ramsoy, T.Z. (2013) Global Workspace Dynamics: Cortical "Binding and Propagation" Enables Conscious Contents. Frontiers in Psychology, 4, 200. https://doi.org/10.3389/fpsyg.2013.00200

[47] Oizumi, M., Albantakis, L. and Tononi, G. (2014) From the Phenomenology to the Mechanisms of Consciousness: Integrated Information Theory 3.0. PLOS Computational Biology, 10, e1003588. https://doi.org/10.1371/journal.pcbi.1003588

[48] Tononi, G. (2008) Consciousness as Integrated Information: A Provisional Manifesto. The Biological Bulletin, 215, 216-242. https://doi.org/10.2307/25470707

[49] Blackmore, S. and Troscianko, E. (2018) Consciousness: An Introduction. Routledge, Abingdon-on-Thames.

[50] Tononi, G. and Edelman, D.B. (1998) Consciousness and Complexity. Science, 282, 1846-1851.

[51] Edelman, G. (1987) Neural Darwinism. Basic Books, New York. 
[52] Edelman, G.M. (2003) Naturalizing Consciousness: A Theoretical Framework. Proceedings of the National Academy of Sciences, 100, 5520-5524. https://doi.org/10.1073/pnas.0931349100

[53] Metzinger, T. (2000) Neural Correlates of Consciousness: Empirical and Conceptual Questions. MIT Press, Bradford.

[54] Tononi, G. and Edelman, G.M. (2000) A Universe of Consciousness: How Matter Becomes Imagination. Basic Books, New York.

[55] Srinivasan, R., et al. (1999) Increased Synchronization of Neuromagnetic Responses during Conscious Perception. The Journal of Neuroscience, 19, 5435-5448. https://doi.org/10.1523/JNEUROSCI.19-13-05435.1999

[56] Driver, J. and Vuilleumier, P. (2001) Perceptual Awareness and Its Loss in Unilateral Neglect and Extinction. Cognition, 79, 39-88. https://doi.org/10.1016/S0010-0277(00)00124-4

[57] Luukkainen-Markkula, R., et al. (2011) Hemispatial Neglect Reflected on Visual Memory. Restorative Neurology and Neuroscience, 29, 321-330.

[58] Sacks, O. (1985) The Man Who Mistook His Wife for a Hat. Picador, London.

[59] Jerath, R., et al. (2015) Etiology of Phantom Limb Syndrome: Insights from a 3D Default Space Consciousness Model. Medical Hypotheses, 85, 153-159.

https://doi.org/10.1016/j.mehy.2015.04.025

[60] Snyder, F. (1970) The Phenomenology of Dreaming. In: Madow, L. and Snow, L.H., Eds., The Psychodynamic Implications of the Physiological Studies on Dreams, Charles S Thomas, Springfield, 124-151.

[61] Foulkes, D. (1985) Dreaming: A Cognitive-Psychological Analysis. Lawrence Erlbaum, Hillsdale.

[62] Jerath, R., et al. (2016) Functional and Neural Mechanisms of Out-of-Body Experiences: Importance of Retinogeniculo-Cortical Oscillations. World Journal of Neuroscience, 6, 287-302. https://doi.org/10.4236/wjns.2016.64032

[63] Fingelkurts, A.A., et al. (2003) Cortical Operational Synchrony during Audio-Visual Speech Integration. Brain and Language, 85, 297-312.

https://doi.org/10.1016/S0093-934X(03)00059-2

[64] Engel, A.K. and Singer, W. (2001) Temporal Binding and the Neural Correlates of Sensory Awareness. Trends in Cognitive Sciences, 5, 16-25. https://doi.org/10.1016/S1364-6613(00)01568-0

[65] Calvert, G.A., et al. (2001) Detection of Audio-Visual Integration Sites in Humans by Application of Electrophysiological Criteria to the BOLD Effect. NeuroImage, 14, 427-438. https://doi.org/10.1006/nimg.2001.0812

[66] Steriade, M. (2006) Grouping of Brain Rhythms in Corticothalamic Systems. Neuroscience, 137, 1087-1106. https://doi.org/10.1016/j.neuroscience.2005.10.029

[67] Honey, C.J., et al. (2009) Predicting Human Resting-State Functional Connectivity from Structural Connectivity. Proceedings of the National Academy of Sciences, 106, 2035-2040. https://doi.org/10.1073/pnas.0811168106

[68] Izhikevich, E.M. and Edelman, G.M. (2008) Large-Scale Model of Mammalian Thalamocortical Systems. Proceedings of the National Academy of Sciences of the United States of America, 105, 3593-3598. https://doi.org/10.1073/pnas.0712231105

[69] Jones, E.G. (1985) The Thalamus. Plenum, New York. https://doi.org/10.1007/978-1-4615-1749-8

[70] Gollo, L.L., Mirasso, C. and Villa, A.E.P. (2010) Dynamic Control for Synchroniza- 
tion of Separated Cortical Areas through Thalamic Relay. NeuroImage, 52, 947-955. https://doi.org/10.1016/j.neuroimage.2009.11.058

[71] Edelman, G.M. and Gally, J.A. (2013) Reentry: A Key Mechanism for Integration of Brain Function. Frontiers in Integrative Neuroscience, 7, 63.

https://doi.org/10.3389/fnint.2013.00063

[72] Andrews-Hanna, J.R., Smallwood, J. and Spreng, R.N. (2014) The Default Network and Self-Generated Thought: Component Processes, Dynamic Control, and Clinical Relevance. Annals of the New York Academy of Sciences, 1316, 29-52. https://doi.org/10.1111/nyas.12360

[73] Andrews-Hanna, J.R. (2012) The Brain's Default Network and Its Adaptive Role in Internal Mentation. The Neuroscientist. A Review Journal Bringing Neurobiology, Neurology and Psychiatry, 18, 251-270. https://doi.org/10.1177/1073858411403316

[74] Gusnard, D.A., et al. (2001) Medial Prefrontal Cortex and Self-Referential Mental Activity: Relation to a Default Mode of Brain Function. Proceedings of the National Academy of Sciences, 98, 4259-4264. https://doi.org/10.1073/pnas.071043098

[75] Gusnard, D.A. (2005) Being a Self: Considerations from Functional Imaging. Consciousness and Cognition, 14, 679-697. https://doi.org/10.1016/j.concog.2005.04.004

[76] Fingelkurts, A., et al. (2003) Structural (Operational) Synchrony of EEG Alpha Activity during an Auditory Memory Task. NeuroImage, 20, 529-542. https://doi.org/10.1016/S1053-8119(03)00305-7

[77] Fingelkurts, A.A. and Fingelkurts, A.A. (2011) Persistent Operational Synchrony within Brain Default-Mode Network and Self-Processing Operations in Healthy Subjects. Brain and Cognition, 75, 79-90.

https://doi.org/10.1016/j.bandc.2010.11.015

[78] Tong, Y., et al. (2013) Evaluating the Effects of Systemic Low Frequency Oscillations Measured in the Periphery on the Independent Component Analysis Results of Resting State Networks. NeuroImage, 76, 202-215.

https://doi.org/10.1016/j.neuroimage.2013.03.019

[79] Schilbach, L., et al. (2008) Minds at Rest? Social Cognition as the Default Mode of Cognizing and Its Putative Relationship to the "Default System" of the Brain. Consciousness and Cognition, 17, 457-467. https://doi.org/10.1016/j.concog.2008.03.013

[80] Fingelkurts, A.A., Fingelkurts, A.A. and Kallio-Tamminen (2016) Long-Term Meditation Training Induced Changes in the Operational Synchrony of Default Mode Network Modules during a Resting State. Cognitive Processing, 17, 27-37. https://doi.org/10.1007/s10339-015-0743-4

[81] Musholt, K. (2013) A Philosophical Perspective on the Relation between Cortical Midline Structures and the Self. Frontiers in Human Neuroscience, 7, 536. https://doi.org/10.3389/fnhum.2013.00536

[82] Moran, J., Kelley, W. and Heatherton, T. (2013) What Can the Organization of the Brain's Default Mode Network Tell Us About Self-Knowledge? Frontiers in Human Neuroscience, 7, 391. https://doi.org/10.3389/fnhum.2013.00391

[83] Kerr, C.E., Josyula, K. and Littenberg, R. (2011) Developing an Observing Attitude: An Analysis of Meditation Diaries in an MBSR Clinical Trial. Clinical Psychology \& Psychotherapy, 18, 80-93. https://doi.org/10.1002/cpp.700

[84] Newberg, A.B. and Iversen, J. (2003) The Neural Basis of the Complex Mental Task of Meditation: Neurotransmitter and Neurochemical Considerations. Medical Hypotheses, 61, 282-291. https://doi.org/10.1016/S0306-9877(03)00175-0

[85] Newberg, A., et al. (2001) The Measurement of Regional Cerebral Blood Flow dur- 
ing the Complex Cognitive Task of Meditation: A Preliminary SPECT Study. Psychiatry Research: Neuroimaging, 106, 113-122. https://doi.org/10.1016/S0925-4927(01)00074-9

[86] Jerath, R., et al. (2012) Dynamic Change of Awareness during Meditation Techniques: Neural and Physiological Correlates. Frontiers in Human Neuroscience, 6, 131. https://doi.org/10.3389/fnhum.2012.00131

[87] Lakatos, P. (2005) An Oscillatory Hierarchy Controlling Neuronal Excitability and Stimulus Processing in the Auditory Cortex. Journal of Neurophysiology, 94, 1904-1911. https://doi.org/10.1152/jn.00263.2005

[88] Herrero, J.L., et al. (2018) Breathing above the Brain Stem: Volitional Control and Attentional Modulation in Humans. Journal of Neurophysiology, 119, 145-159. https://doi.org/10.1152/jn.00551.2017

[89] Jerath, R. and Crawford, M.W. (2014) Neural Correlates of Visuospatial Consciousness in 3D Default Space: Insights from Contralateral Neglect Syndrome. Consciousness and Cognition, 28, 81-93. https://doi.org/10.1016/j.concog.2014.06.008

[90] Prakash, R., et al. (2008) Global Workspace Model of Consciousness and Its Electromagnetic Correlates. Annals of Indian Academy of Neurology, 11, 146-153. https://doi.org/10.4103/0972-2327.42933

[91] Kemmerer, D. (2015) Are We Ever Aware of Concepts? A Critical Question for the Global Neuronal Workspace, Integrated Information, and Attended Intermediate-Level Representation Theories of Consciousness. Neuroscience of Consciousness, 2015, niv006.

[92] Tononi, G., et al. (2016) Integrated Information Theory: From Consciousness to Its Physical Substrate. Nature Reviews Neuroscience, 17, 450-461. https://doi.org/10.1038/nrn.2016.44

[93] Steriade, M. (2003) The Corticothalamic System in Sleep. Frontiers in Bioscience, 8, d878-d899. https://doi.org/10.2741/1043

[94] Magnin, M., et al. (2010) Thalamic Deactivation at Sleep Onset Precedes That of the Cerebral Cortex in Humans. Proceedings of the National Academy of Sciences, 107, 3829-3833. https://doi.org/10.1073/pnas.0909710107

[95] Eagleman, D. (2011) Incognito: The Secret Lives of the Brain. Pantheon.

[96] Bargh, J.A. and Morsella, E. (2008) The Unconscious Mind. Perspectives on Psychological Science: A Journal of the Association for Psychological Science, 3, 73-79. https://doi.org/10.1111/j.1745-6916.2008.00064.x

[97] Trehub, A. (2007) Space, Self, and the Theater of Consciousness. Consciousness and Cognition, 16, 310-330. https://doi.org/10.1016/j.concog.2006.06.004

[98] Craik, K. (1943) The Nature of Explanation. Cambridge University Press, Cambridge.

[99] Revonsuo, A. (2006) Inner Presence: Consciousness as a Biological Phenomenon. MIT Press, Cambridge.

[100] Metzinger, T. (2003) Being No-One. MIT Press, Cambridge.

[101] Grush, R. (2004) The Emulation Theory of Representation: Motor Control, Imagery, and Perception. Behavioral and Brain Sciences, 27, 377-442.

https://doi.org/10.1017/S0140525X04000093

[102] Jerath, R., et al. (2016) Meditation Experiences, Self, and Boundaries of Consciousness. International Journal of Complementary \& Alternative Medicine, 4, Article 
ID: 00105 .

[103] Sullivan, P.R. (1995) Contentless Consciousness and Information-Processing Theories of Mind. Philosophy, Psychiatry \& Psychology, 2, 51-59.

[104] Colby, C.L. and Goldberg, M.E. (1999) Space and Attention in Parietal Cortex. Annual Review of Neuroscience, 22, 319-349. https://doi.org/10.1146/annurev.neuro.22.1.319

[105] Goldberg, M.E., et al. (2006) Saccades, Salience and Attention: The Role of the Lateral Intraparietal Area in Visual Behavior. Progress in Brain Research, 155, 157-175. https://doi.org/10.1016/S0079-6123(06)55010-1

[106] McGlinchey-Berroth, R., et al. (1996) Semantic Processing and Orthographic Specificity in Hemispatial Neglect. Journal of Cognitive Neuroscience, 8, 291-304. https://doi.org/10.1162/jocn.1996.8.3.291

[107] Celesia, G.G. (2010) Visual Perception and Awareness. Journal of Psychophysiolo$g y$, 24, 62-67. https://doi.org/10.1027/0269-8803/a000014

[108] Persaud, N. and Cowey, A. (2008) Blindsight Is Unlike Normal Conscious Vision: Evidence from an Exclusion Task. Consciousness and Cognition, 17, 1050-1055. https://doi.org/10.1016/j.concog.2007.10.002

[109] Horgan, J. (2015) Can Integrated Information Theory Explain Consciousness? Scientific American.

[110] Tononi, G. (2014) Why Scott Should Stare at a Blank Wall and Reconsider (or, the Conscious Grid). The Blog of Scott Aaronson.

[111] Edelman, G. and Tononi, G. (2008) A Universe of Consciousness: How Matter Becomes Imagination. Basic Books, New York.

[112] Emadi, N., Rajimehr, R. and Esteky, H. (2014) High Baseline Activity in Inferior Temporal Cortex Improves Neural and Behavioral Discriminability during Visual Categorization. Frontiers in Systems Neuroscience, 8, 218. https://doi.org/10.3389/fnsys.2014.00218

[113] Cohen, M.A. and Dennett, D.C. (2011) Consciousness Cannot Be Separated from Function. Trends in Cognitive Sciences, 15, 358-364. https://doi.org/10.1016/j.tics.2011.06.008

[114] von Arx, S.W., et al. (2010) Anosognosia for Cerebral Achromatopsia-A Longitudinal Case Study. Neuropsychologia, 48, 970-977. https://doi.org/10.1016/j.neuropsychologia.2009.11.018

[115] Jerath, R. and Crawford, M.W. (2015) How Does the Body Affect the Mind? Role of Cardiorespiratory Coherence in Spectrum of Emotions. Advances in Mind-Body Medicine, 29, 4-16.

[116] Aftanas, L.I., et al. (2004) Analysis of Evoked EEG Synchronization and Desynchronization in Conditions of Emotional Activation in Humans: Temporal and Topographic Characteristics. Neuroscience and Behavioral Physiology, 34, 859-867. https://doi.org/10.1023/B:NEAB.0000038139.39812.eb

[117] Bosse, T., Jonker, C.M. and Treur, J. (2008) Formalization of Damasio's Theory of Emotion, Feeling and Core Consciousness. Consciousness and Cognition, 17, 94-113. https://doi.org/10.1016/j.concog.2007.06.006

[118] Murphy, N., Brown, W.S. and Press, O.U. (2007) Did My Neurons Make Me Do It? Philosophical and Neurobiological Perspectives on Moral Responsibility and Free Will. Clarendon Press. https://doi.org/10.1093/acprof:oso/9780199215393.001.0001

[119] Hyafil, A., et al. (2015) Neural Cross-Frequency Coupling: Connecting Architec- 
tures, Mechanisms, and Functions. Trends in Neurosciences, 38, 725-740. https://doi.org/10.1016/j.tins.2015.09.001

[120] Tort, A.B.L., Brankačk, J. and Draguhn, A. (2018) Respiration-Entrained Brain Rhythms Are Global But Often Overlooked. Trends in Neurosciences, 41, 186-197. https://doi.org/10.1016/j.tins.2018.01.007 